\title{
Mechanistic Studies of Liquid Metal Anode SOFCs II: Development of a coulometric technique to provide important additional insight and aid reactor design
}

\author{
Aliya Toleuova, ${ }^{\mathrm{a}, \mathrm{b}}$ William C. Maskell, ${ }^{\mathrm{a}, \mathrm{c}^{*}}$ Vladimir Yufit, ${ }^{\mathrm{c}}$ Paul R. Shearing ${ }^{\mathrm{a}}$ and Daniel \\ J.L. Brett ${ }^{\mathrm{a}^{*}}$ \\ ${ }^{a}$ Electrochemical Innovation Lab, Department of Chemical Engineering, University College \\ London, WC1E 7JE, London, UK \\ ${ }^{b}$ School of Engineering, Nazarbayev University, 010000, Astana, Kazakhstan \\ ${ }^{c}$ Department of Earth Science and Engineering, Imperial College London, London, SW7 2AZ, \\ $U K$ \\ *Authors to whom correspondence should be addressed: w.maskell@ucl.ac.uk; \\ d.brett@ucl.ac.uk, +44(0)2076793810
}

\section{Abstract}

Improved understanding of the operation of liquid metal anode solid oxide fuel cells (LMA-SOFCs) is required to progress this promising energy conversion technology. In order to facilitate analysis and interpretation, initial studies have been carried out with a simple system in which hydrogen is used as the fuel and the liquid metal electrode is operated in a potential region where it effectively behaves as an 'inert' solvent for dissolved gases. A model for the processes taking place in a liquid tin anode (LTA) supplied with hydrogen has previously been reported which identified a key parameter, the Dynamic Oxygen Utilisation Coefficient, $\bar{z}$, important for understanding the operation and design of these systems. This parameter serves a similar role to the Damköhler number, widely applied in chemical reaction engineering to relate the chemical reaction rate to the transport phenomena rate. This paper describes the development of a method, named Anodic Injection Coulometry (AIC), to determine $\bar{z}$, together with an example of its application. 


\section{Keywords}

Anodic injection coulometry; liquid tin; hydrogen; solid oxide fuel cell; molten metal anode.

\section{Introduction}

Solid oxide fuel cells (SOFCs) with liquid metal anodes (LMAs) have substantial potential for power generation. The advantages over combustion systems and other types of fuel cell have been reviewed. ${ }^{1}$ The technology is able to provide high efficiency and low environmental impact with flexibility regarding fuels, including solid fuel such as coal. ${ }^{2}$ Development of this promising technology, including scale up to pilot plant and industrial systems, requires an in-depth understanding of the mechanism of operation and solutions to a number of technological challenges.

In order to facilitate analysis and interpretation, initial studies have been carried out with a simple system in which hydrogen is used as the fuel and the liquid metal electrode is operated in a potential region where it effectively behaves as an 'inert' solvent for dissolved gases. The previous paper (Part I) described the fundamental electrochemistry of such a LMA SOFC system. ${ }^{3}$ It was demonstrated that the oxidation of hydrogen takes place via a ChemicalElectrochemical mechanism, (so-called CE mode), whereby hydrogen undergoes fast dissolution, then rate-determining homogeneous oxidation by oxygen dissolved in the liquid tin, followed by diffusion-controlled anodic oxygen injection to replace oxygen removed by the chemical reaction. In summary, the process involves:

- Fast dissolution of hydrogen via Sievert's law ${ }^{4-7}$ :

$$
H_{2} \leftrightarrows 2[H]_{S n}
$$

- Homogeneous (rate-determining) oxidation of dissolved hydrogen by dissolved oxygen: 


$$
2[\mathrm{H}]_{S n}+[\mathrm{O}]_{S n} \rightarrow \mathrm{H}_{2} \mathrm{O}
$$

- Anodic injection of oxygen to replace that consumed in (2):

$$
\left[O^{2-}\right]_{Y S Z} \leftrightarrows[O]_{S n}+2 e^{-}
$$

The oxygen injected diffuses into the bulk of the tin from the interface.

The assumptions made in developing the model were as follows:

i) The electrochemical kinetics of the electrode reaction are fast, so that the concentration of dissolved oxygen at the YSZ-tin interface is fixed by the applied potential and is not disturbed by the current flow.

ii) The concentration of dissolved hydrogen in liquid tin is the saturated concentration appropriate to the partial pressure of the hydrogen supplied (bubbled) as a gas and obeys Sievert's law.

iii) Homogeneous oxidation of hydrogen by dissolved oxygen is the rate-determining step and is governed by a rate equation which is first-order with respect to monatomic oxygen.

iv) Under potentiostatic control, diffusion of oxygen away from the YSZ-tin interface to replace that removed by reaction with hydrogen occurs through a diffusion layer of constant thickness, $\delta$, as a result of convection induced by bubbling and thermal effects.

v) The system is in quasi-equilibrium.

Consider the following: hydrogen is bubbled through the liquid tin at partial pressure $p_{1}$, a potential, $E$, is applied to the Sn / YSZ interface and eventually a steady current, $i_{l}$, is observed. The partial pressure of $\mathrm{H}_{2}$ is then increased to $p_{2}$ ' and the current, $i$, increases 
with time and eventually stabilises at $i_{2}$ (Figure 1). Oxygen concentrations within the liquid tin are described schematically in Figure 2.

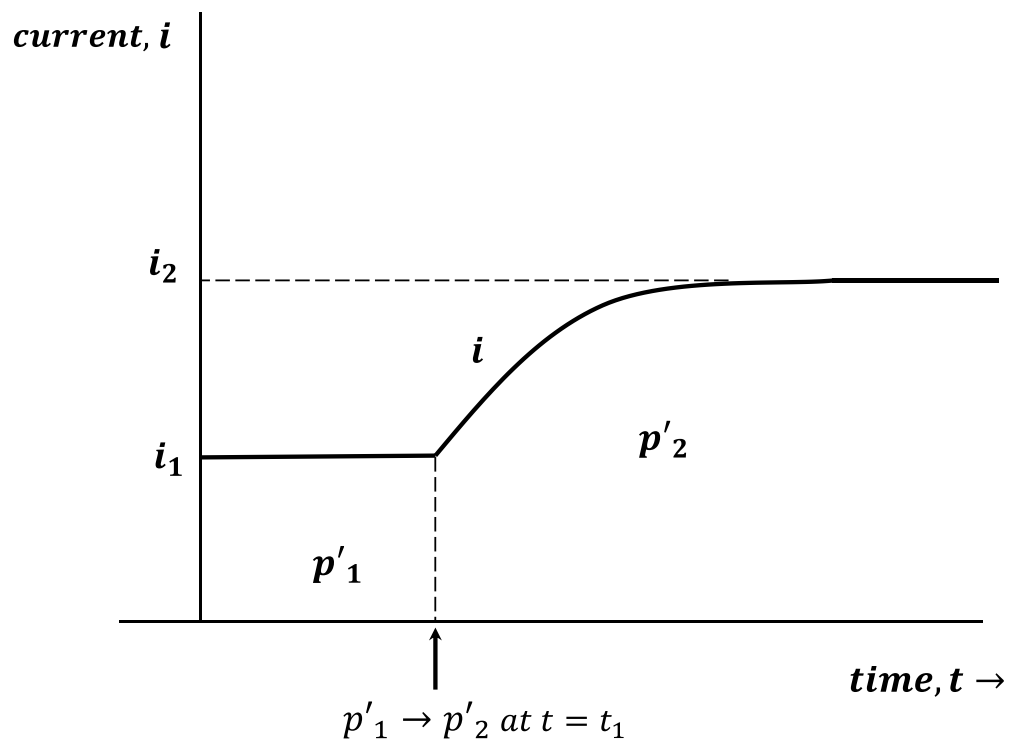

Figure 1. Schematic showing the step change in $p\left(\mathrm{H}_{2}\right)$ which results in additional anodic injection of oxygen at a given applied potential in the $C E$ mode of operation.

In the theoretical treatment, hydrogen concentration is denoted by a single prime and oxygen concentration is denoted by a bar.

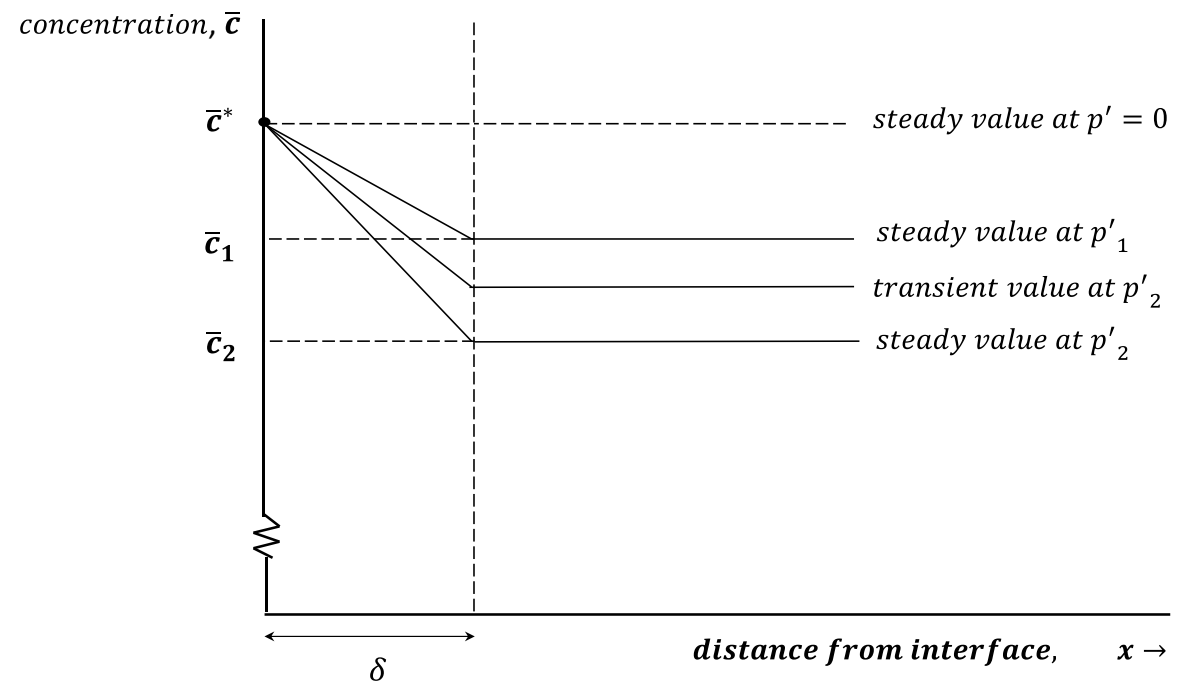

Figure 2. Schematic of the dissolved oxygen concentration profile at a given applied potential in the $C E$ mode of operation. The diffusion layer thickness is shown as $\delta$. 
The rate of removal of oxygen via Reaction (2) is $V k_{1} \bar{c}\left(c^{\prime}\right)^{2}$, (i.e. $k_{1} \bar{c}\left(c^{\prime}\right)^{2}$ per unit volume of tin) where $k_{l}$ is the rate constant for the reaction and $V$ is the volume of tin.

The rate of injection of oxygen via Reaction (3) is $i / n F(n=2)$. Thus the net rate of removal of oxygen via the two reactions is $V k_{1} \bar{c}\left(c^{\prime}\right)^{2}-i / n F$. The concentration of hydrogen, $c^{\prime}$, for given hydrogen partial pressure, $p$ 'is given by (according to Sievert's law):

$$
c^{\prime}=k_{2}\left(p^{\prime}\right)^{1 / 2}
$$

where $k_{2}$ is a constant for a given temperature (Sievert's constant).

Expressing the total amount (gram atoms) of [O] in tin as $\bar{N}$, it follows that

$$
\frac{d \bar{N}}{d t}=-V k_{1}\left(k_{2}\right)^{2} \bar{c} p^{\prime}+\frac{i}{n F}
$$

Applying Fick's first law to the diffusion of [O] from the interface, the flux, $\bar{J}$, is given by:

$$
\bar{J}=-\bar{D} A \frac{d \bar{c}}{d x}
$$

where $\bar{D}$ is the diffusion coefficient, $A$ is the area of the interface and $\frac{d \bar{c}}{d x}$ is the concentration gradient within the diffusion layer. So Equation (6) becomes (see Figure 2):

$$
\bar{J}=-\bar{D} A\left(\frac{\bar{c}-\bar{c}^{*}}{\delta}\right)
$$

Equating the flux with current via Faraday's law:

$$
\bar{J}=\frac{i}{n F}
$$

Eliminating $\bar{J}$ between (7) and (8):

$$
i=\frac{n F \bar{D} A}{\delta}\left(\bar{c}^{*}-\bar{c}\right)
$$


This will be written as:

$$
i=k_{3}\left(\bar{c}^{*}-\bar{c}\right)
$$

where $k_{3}$ is given by:

$$
k_{3}=\frac{n F \bar{D} A}{\delta}
$$

In the steady-state situation, we have $p^{\prime}=p_{2}^{\prime}, \bar{c}=\bar{c}_{2}, i=i_{2}$ and $\frac{d \bar{N}}{d t}=0$.

So using Equation (5):

$$
-V k_{1}\left(k_{2}\right)^{2} \bar{c}_{2} p_{2}^{\prime}=\frac{i_{2}}{n F}
$$

And from (10)

$$
i_{2}=k_{3}\left(\bar{c}^{*}-\bar{c}_{2}\right)
$$

Eliminating $i_{2}$ between (12) and (13) and rearranging:

$$
\bar{c}_{2}=\bar{c}^{*}\left(1+\frac{V k_{1}\left(k_{2}\right)^{2} p_{2}^{\prime} n F}{k_{3}}\right)^{-1}
$$

Writing

$$
\bar{z}_{2}=\left(1+\frac{V k_{1}\left(k_{2}\right)^{2} p_{2}^{\prime} n F}{k_{3}}\right)^{-1}
$$

Then

$$
\bar{c}_{2}=\bar{z}_{2} \bar{c}^{*}
$$

Note that $\bar{z}$ is a function of $p$ and $\bar{z} \leq 1$. Likewise:

$$
\bar{c}_{1}=\bar{z}_{1} \bar{c}^{*}
$$

We may write: 


$$
\frac{d \bar{c}}{d t}=\frac{1}{V} \frac{d \bar{N}}{d t}
$$

Eliminating $i$ between (5) and (10) for $p^{\prime}=p_{2}^{\prime}$

$$
\frac{d \bar{N}}{d t}=-V k_{1}\left(k_{2}\right)^{2} \bar{c} p_{2}{ }^{\prime}+\frac{k_{3}\left(\bar{c}^{*}-\bar{c}\right)}{n F}
$$

From (18) and (19):

$$
\frac{d \bar{c}}{d t}=\frac{k_{3}}{V n F}\left[-\bar{c}\left(\frac{n F V k_{1}\left(k_{2}\right)^{2} p_{2}{ }^{\prime}}{k_{3}}+1\right)+\bar{c}^{*}\right]
$$

Then using Equation (15)

$$
\frac{d \bar{c}}{d t}=\frac{k_{3}}{V n F \bar{z}_{2}}\left(-\bar{c}+\bar{z}_{2} \bar{c}^{*}\right)
$$

Followed by (16):

$$
\frac{d \bar{c}}{d t}=\frac{k_{3}}{V n F \bar{z}_{2}}\left(\bar{c}_{2}-\bar{c}\right)
$$

Integrating:

$$
\begin{gathered}
\int_{\bar{c}_{1}}^{\bar{c}} \frac{d \bar{c}}{\left(\bar{c}_{2}-\bar{c}\right)}=\frac{k_{3}}{V n F \bar{z}_{2}} \int_{t_{1}}^{t} d t \\
-\ln \left[\bar{c}_{2}-\bar{c}\right]_{\bar{c}_{1}}^{\bar{c}}=\frac{k_{3}}{V n F \bar{z}_{2}}\left(t-t_{1}\right) \\
\ln \frac{\bar{c}_{2}-\bar{c}}{\bar{c}_{2}-\bar{c}_{1}}=-\frac{k_{3}}{V n F \bar{z}_{2}}\left(t-t_{1}\right)
\end{gathered}
$$

And from (12) and (16):

$$
i_{2}=n F V k_{1}\left(k_{2}\right)^{2} \bar{z}_{2} \bar{c}^{*} p_{2}^{\prime}
$$




\section{Dynamic Oxygen Utilisation Coefficient as a characteristic cell parameter for LTA SOFCs}

A new key parameter $(\bar{z})$, herewith termed the Dynamic Oxygen Utilisation Coefficient, has evolved during the model development, the value of which is important for LMA-SOFC design.

The coefficient $(\bar{z})$ is defined by Equation (15) above and contains the dimensionless group, $\frac{V k_{1}\left(k_{2}\right)^{2} p_{2}^{\prime} n F}{k_{3}}$ with similarity to the Damköhler number, which relates the chemical reaction timescale to the transport phenomena rate occurring in a system. ${ }^{8,9}$

The general definition of Damköhler number, Da, is given by the following ratio9:

$$
\mathrm{Da}=\frac{\tau_{C}}{\tau_{M}}
$$

where $\tau_{C}$ is the characteristic time of the chemical reaction and $\tau_{M}$ is the mixing time that can be controlled by hydrodynamics. In systems that include interphase mass-transfer (via diffusion), a second Damköhler number, Da

$$
\mathrm{Da}_{\mathrm{II}}=\frac{k_{w}}{k_{f} a_{s}}
$$

Where $k_{w}$ is the rate coefficient in $\mathrm{m}^{3}\left(\mathrm{~kg}_{\mathrm{cat}}\right)^{-1} \mathrm{~s}^{-1}, k_{f}$ is the mass-transfer coefficient in $\mathrm{m} \mathrm{s}^{-1}$ and $a_{s}$ is the specific external surface area of the reacting substrate in $\mathrm{m}^{2}\left(\mathrm{~kg}_{\mathrm{cat}}\right)^{-1}$.

The dimensionless group, $\frac{V k_{1}\left(k_{2}\right)^{2} p_{2}^{\prime} n F}{k_{3}}$, together with Equation (11) can be represented by the expression $\frac{V k_{1}\left(k_{2}\right)^{2} \delta p_{2}^{\prime}}{A D}$. At a given temperature, this term is dependent upon geometric factors $(V / A)$, mass transfer factors (via $\delta / D$ which decreases with increasing degree of convection), and a kinetic factor $\left(k_{1}\right)$, complementary to the factors $a_{s}, k_{f}$ and $k_{w}$ respectively in the definition of DaII (see above). 
The value of $\bar{z}$ determines the output current of the cell, as shown in Equation (26). The larger the value of $k_{3}$ (i.e. large $A$; small $\delta$ ) then $\bar{Z}$ tends towards unity; conversely, the smaller the value of $k_{3}$ (i.e. small $A$; large $\delta$ ) then $\bar{Z}$ tends towards small values with a limiting value of zero.

In this paper, a new method is proposed, namely Anodic Injection Coulometry (AIC), similar in principle to the well-known technique of anodic stripping voltammetry (ASV) ${ }^{11}$, to determine $\bar{z}$. To this end, two related regimes of operation are proposed. These regimes are run in a mixed mode (i.e. potentiostatic and open-circuit), whereby electrode potential is switched between a fixed value $E$ and open-circuit. During the open-circuit periods, hydrogen reacts with oxygen dissolved in tin according to a Chemical-Electrochemical (CE) mechanism ${ }^{3}$. In the first regime (Regime 1) the flow of $\mathrm{H}_{2}$ into the tin is constant, whereas in the second (Regime 2) it is intermittent. This method applies theory developed specifically for each regime. An example of the application of this method for measurement of $\bar{z}$ is presented.

\section{Experimental}

The work employed a SOFC with liquid tin anode. A schematic of the apparatus employed in this work is shown in Figure 3 and is described as follows. Dry argon was supplied to the oxygen sensor (Kent Industrial Measurements, UK) and then downstream was mixed with hydrogen (both gases were zero grade, BOC, UK) prior to being supplied to the cell (Figure 4) held at operating temperature $\left(780^{\circ} \mathrm{C}\right)$ by an electric furnace (Carbolite, UK). Gases entered and exited the cell via alumina tubes, the lower end of the inlet tube being immersed in the working electrode (WE) so that the gas bubbled through the liquid tin. Gas flows were maintained at specified flow rates (up to $100 \mathrm{ml}$ per minute $\left(1.67 \mathrm{ml} \mathrm{s}^{-1}\right)$ ) with 
EL-FLOW mass flow controllers (Bronkhorst UK Ltd., UK). The WE (25 g of tin shots, 99.99+\%, metal basis) was contained within a closed-end 8YSZ tube (McDanel Advanced Ceramic Technologies, USA) with an internal diameter of $14 \mathrm{~mm}$.

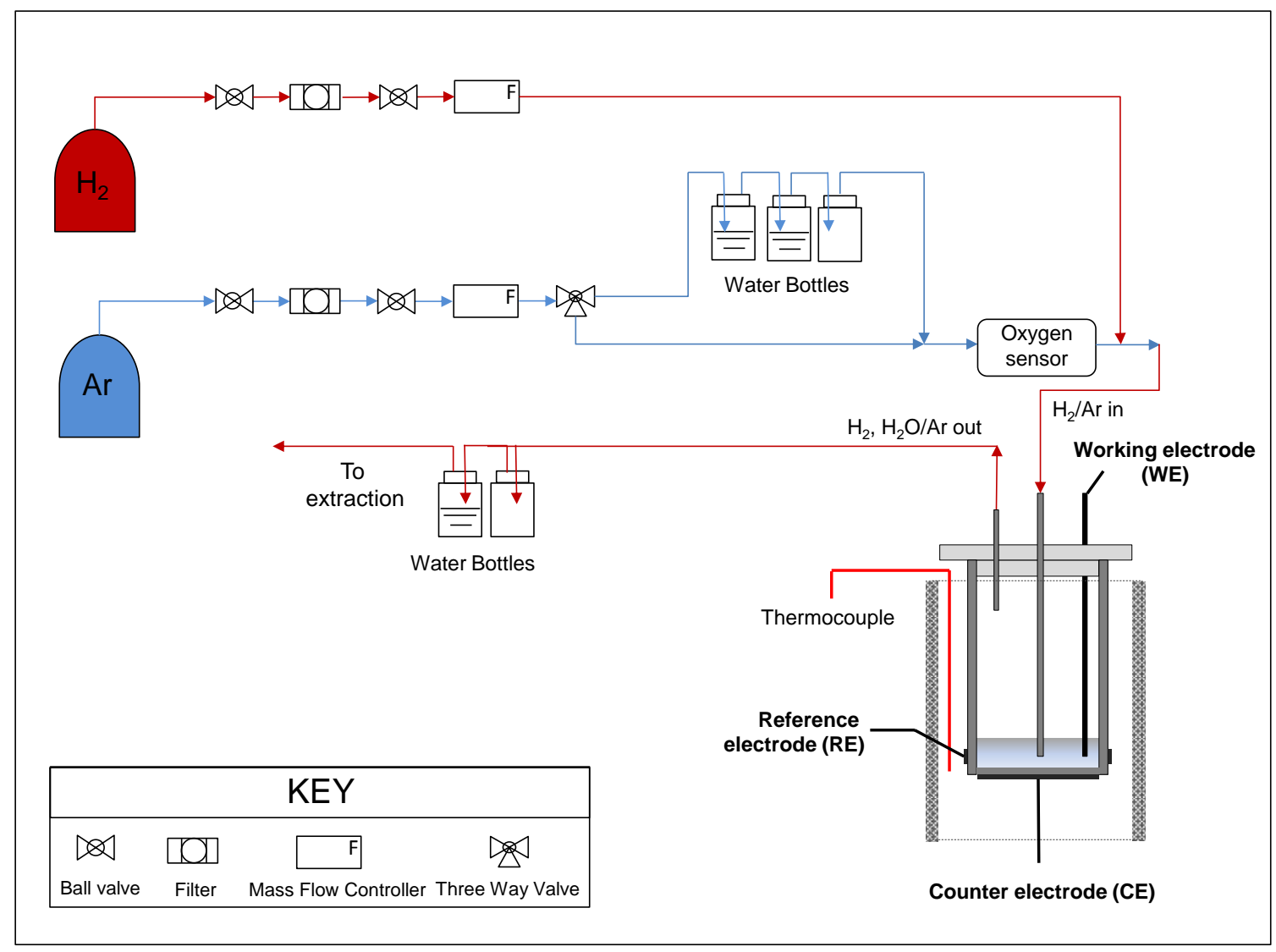

Figure 3 Process flow diagram for oxidation of hydrogen in LMA SOFC cell.

Electrical connection to the WE was achieved using a glassy carbon rod (3 $\mathrm{mm}$ diameter glassy carbon rod - Sigradur HTW Hochtemperatur-Werkstoffe GmbH, Germany) immersed within the liquid tin inside the YSZ tube. Platinum counter and reference electrodes $(4082 \mathrm{Pt}$ Vitr-Au-Less Conductor, Ferro Electronic Materials, USA) were painted on the outer side of the YSZ tube and sintered at $1000{ }^{\circ} \mathrm{C}$ for 2 hours. Current collection from the cathode and reference electrode was achieved using platinum mesh and wires (Goodfellow Cambridge, UK). The total electrode (CE) area was $1.54 \mathrm{~cm}^{2}$. A predrilled pyrophyllite cap (Ceramic Substrates \& Components Ltd., UK) was placed on top of the YSZ tube to hold all tubes and 
the glassy carbon rod in place and sealed with an adhesive (Silicoset 152). Electrochemical measurements were performed using an Ivium potentiostat (Ivium Technologies, Netherlands). The potential of the WE was set at $-0.90 \mathrm{~V}$ versus the air reference which was just within the 'inert' region where the oxygen content of the tin was close to saturation, but there was no formation of tin oxide.

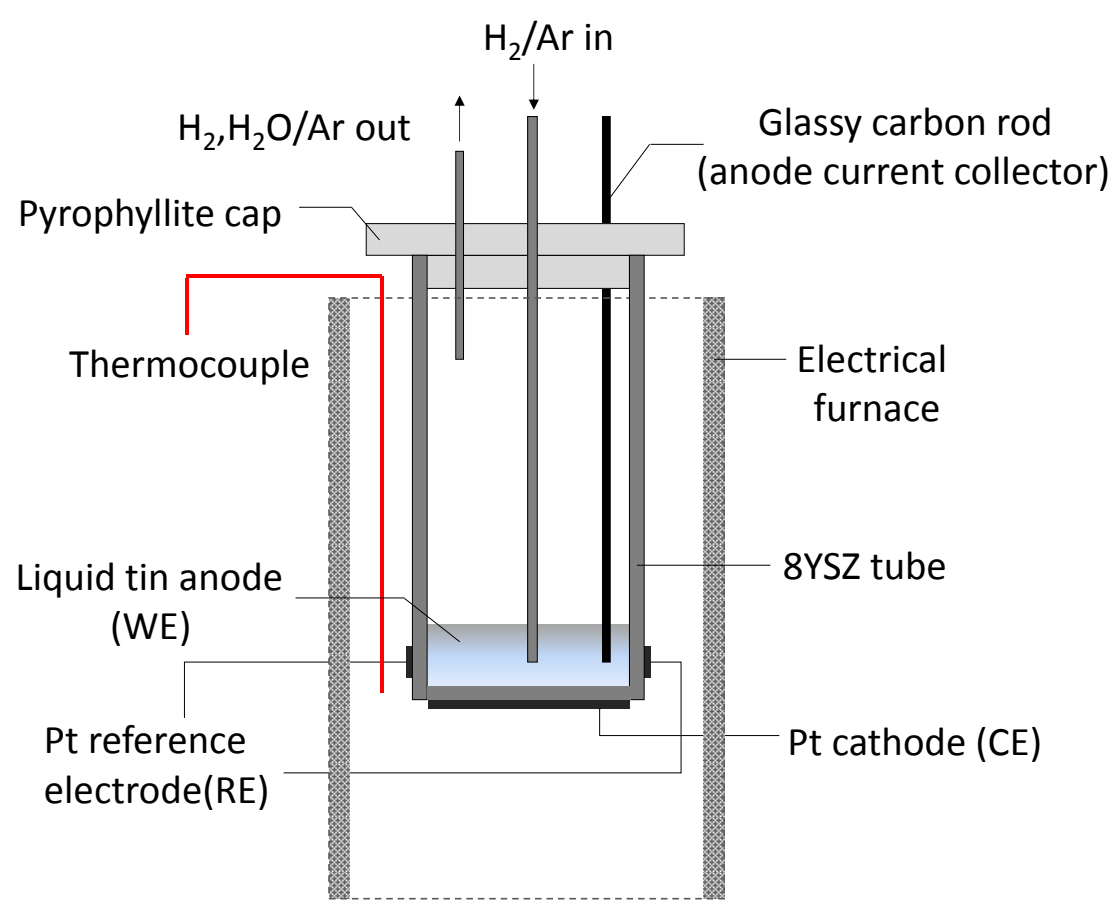

Figure 4 Schematic of the experimental setup of the LMA SOFC cell held in furnace.

\section{Determination of the Dynamic Oxygen Utilisation Coefficient using}

\section{Anodic Injection Coulometry}

In this paper, a technique, namely Anodic Injection Coulometry (AIC) (with similarities to anodic stripping voltammetry), is proposed as a method for determination of the Dynamic Oxygen Utilisation Coefficient, $\bar{z}$, which evolved out of the model for hydrogen oxidation in LTA SOFC operating in the Chemical-Electrochemical mode as shown above. The method proposes AIC applied under two different modes of oxygen injection (termed Regimes 1 and 2). Subsequent analysis of the experimental data in both regimes enables the calculation of $\bar{z}$. 
The determination of the $\bar{Z}$ value is important in order to obtain an understanding of experimentally-generated results. In a broader sense, this parameter is important for the design of systems consisting of two processes: fuel oxidation via dissolved oxygen and oxygen replacement via electrochemical injection at the liquid anode / electrolyte interface. The value of $\bar{z}$ is dictated by the particular geometry and operating conditions of a given cell and hence, requires individual determination in each case. An example is given of how $\bar{z}$ may be determined using AIC.

\section{1. $\quad$ Regime 1}

Consider the following four-stage operation (see Figure 5): prior to Point A, argon is bubbled through liquid tin for a sufficient time to allow the current to reduce to a value close to zero while holding the tin electrode at an anodic potential $E$, typically $-0.90 \mathrm{~V}$ vs. RE (air electrode). It has been shown that this potential results in operation of the system in the $\mathrm{CE}$ mode of operation. ${ }^{3}$ During the intervals A - B, B - C and C - D, hydrogen at partial pressure $p_{2}^{\prime}$ is bubbled through the tin. The interval $\mathrm{A}-\mathrm{B}$ is sufficiently long to allow the current to reach a steady value, $i_{2}$. During the interval B-C, of length $t_{3}-t_{2}$, the tin electrode is switched to open circuit $(\mathrm{OC})$. Then, during the interval $\mathrm{C}-\mathrm{D}$, the potential of the tin electrode is held

at anodic potential $E$, during which time the current is monitored. The period C - D is sufficiently long to allow the current to return to a value close to the steady value, $i_{2}$. The charge, $Q_{1}$, shown as the shaded area in Figure 5 is then calculated. 


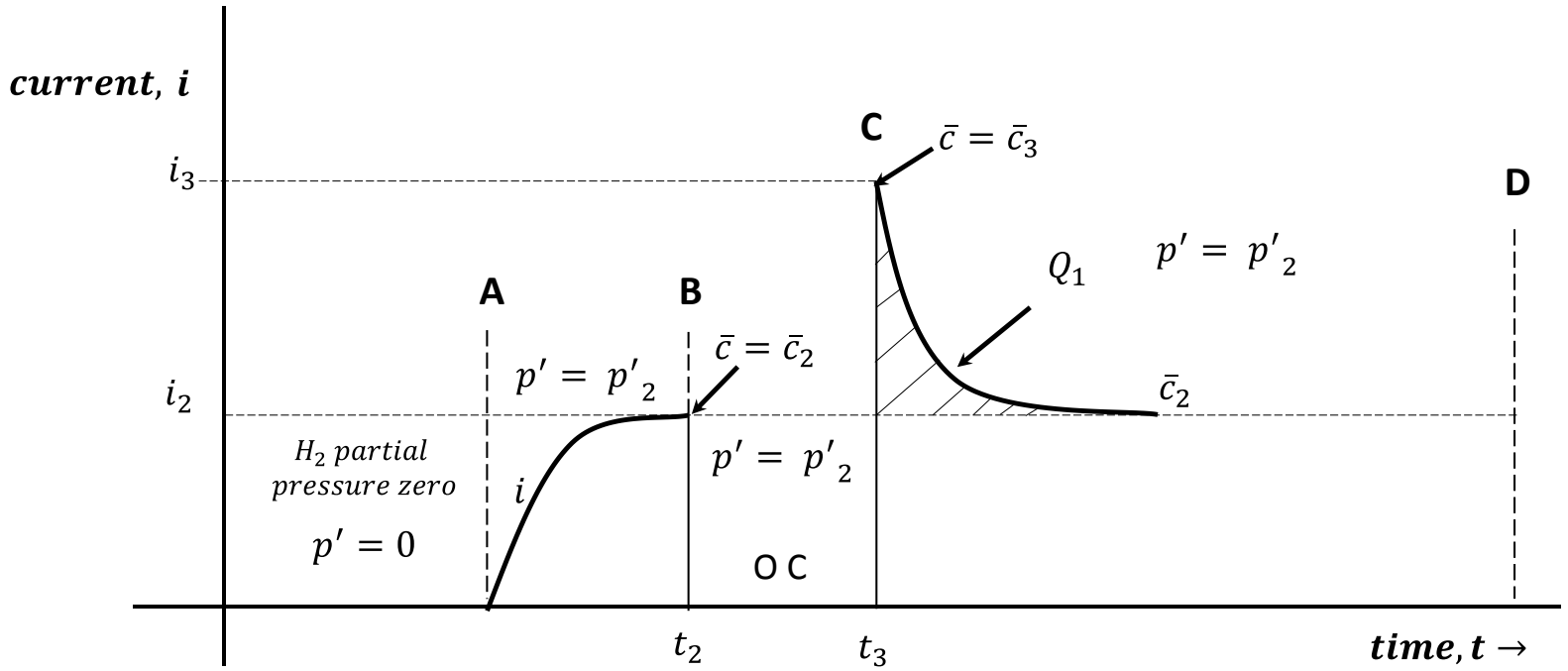

Figure 5 Schematic representation of Regime 1 conditions applied to the working cell: same hydrogen partial pressure from A to $\mathrm{D}, p_{2}$; ; potential is held at a value $E$ everywhere except in B-C.

The interval A-B is treated above. The interval B - C is treated as follows: at open circuit, $i=0$, the equation expressing the rate of removal of oxygen via chemical reaction with hydrogen and the addition of oxygen via anodic injection (Equation (5)) (when treated from $\mathrm{B}-\mathrm{C})$ becomes:

$$
\frac{d \bar{N}}{d t}=-V k_{1}\left(k_{2}\right)^{2} \bar{c} p_{2}{ }^{\prime}
$$

But:

$$
\frac{d \bar{c}}{d t}=\frac{1}{V} \frac{d \bar{N}}{d t}
$$

Using (29) and (18):

$$
\frac{d \bar{c}}{d t}=-k_{1}\left(k_{2}\right)^{2} \bar{c} p_{2}^{\prime}
$$

Integrating (30):

$$
\int_{\bar{c}_{2}}^{\bar{c}_{3}} \frac{d \bar{c}}{\bar{c}}=-k_{1}\left(k_{2}\right)^{2} p_{2}{ }^{\prime} \int_{t_{2}}^{t_{3}} d t
$$


Or

$$
\ln \frac{\bar{c}_{3}}{\bar{c}_{2}}=-k_{1}\left(k_{2}\right)^{2} p_{2}{ }^{\prime}\left(t_{3}-t_{2}\right)
$$

$$
\bar{c}_{3}=\bar{c}_{2} \exp \left[-k_{1}\left(k_{2}\right)^{2} p_{2}{ }^{\prime}\left(t_{3}-t_{2}\right)\right]
$$

The region C-D is now treated, where $p^{\prime}=p_{2}{ }^{\prime}$. The steps used are as follows:

i) The starting value of $\bar{c}$, which is $\bar{c}_{3}$, is given by Equation (32).

ii) An expression is derived for $\bar{c}$ as a function of time.

iii) Using this expression for $\bar{c}$, Equations (10), (13) and (16) reveal the term $\left(i-i_{2}\right)$ as a function of time.

iv) This term $\left(i-i_{2}\right)$ is then integrated from $t_{3}$ to $\infty$ to obtain $Q_{1}$.

Recalling the following equation:

$$
\ln \frac{\bar{c}_{2}-\bar{c}}{\bar{c}_{2}-\bar{c}_{1}}=-\frac{k_{3}}{V n F \bar{z}_{2}}\left(t-t_{1}\right)
$$

Concentration $\bar{c}_{1}$ is replaced by $\bar{c}_{3}$ and $t_{1}$ by $t_{3}$ to obtain:

$$
\bar{c}_{2}-\bar{c}=\left(\bar{c}_{2}-\bar{c}_{3}\right) \exp \left[-\frac{k_{3}}{V n F \bar{z}_{2}}\left(t-t_{3}\right)\right]
$$

From Equations (10) and (13):

$$
i-i_{2}=k_{3}\left(\bar{c}_{2}-\bar{c}\right)
$$

With (33) this leads to:

$$
i-i_{2}=k_{3}\left(\bar{c}_{2}-\bar{c}_{3}\right) \exp \left[-\frac{k_{3}}{V n F \bar{z}_{2}}\left(t-t_{3}\right)\right]
$$

Substituting for $\bar{c}_{3}$ from (32) into (35)and using Equation (16)

$$
i-i_{2}=k_{3} \bar{z}_{2} \bar{c}^{*}\left\{1-\exp \left[-k_{1}\left(k_{2}\right)^{2} p_{2}{ }^{\prime}\left(t_{3}-t_{2}\right)\right]\right\} \times \exp \left[-\frac{k_{3}}{V n F \bar{z}_{2}}\left(t-t_{3}\right)\right]
$$


The charge shown by the shaded area in the diagram (Figure 5) is given by:

$$
\begin{gathered}
Q_{1}=\int_{t_{3}}^{\infty}\left(i-i_{2}\right) d t \\
Q_{1}=-V n F\left(\bar{z}_{2}\right)^{2} \bar{c}^{*}\left\{1-\exp \left[-k_{1}\left(k_{2}\right)^{2} p_{2}{ }^{\prime}\left(t_{3}-t_{2}\right)\right]\right\} \\
\times\left\{\exp \left[-\frac{k_{3}}{V n F \bar{z}_{2}}\left(t-t_{3}\right)\right]\right\}_{t_{3}}^{\infty}
\end{gathered}
$$

Or

$$
Q_{1}=\operatorname{VnF}\left(\bar{z}_{2}\right)^{2} \bar{c}^{*}\left\{1-\exp \left[-k_{1}\left(k_{2}\right)^{2} p_{2}{ }^{\prime}\left(t_{3}-t_{2}\right)\right]\right\}
$$

Equation (39) is the required solution.

\section{Corroborating experimental results in Regime 1}

In order to validate the above theory, the LTA SOFC was operated in Regime 1. After Point A (Figure 5) a mixture of $\mathrm{H}_{2}(16 \mathrm{kPa})$ and $\mathrm{Ar}$ was bubbled continuously through liquid tin, while holding the potential of the WE at $-0.90 \mathrm{~V}$ vs. RE. The partial pressure of $16 \mathrm{kPa}$ hydrogen employed in this study was chosen as representative of the range (0-26 kPa) used in the elucidation of the hydrogen oxidation mechanism in Part I. ${ }^{3}$ Once the anodic current had stabilised at a steady value, the tin electrode was switched to open circuit (OC), remaining so for $1500 \mathrm{~s}$ before controlling the potential again at $-0.90 \mathrm{~V}$ vs. RE. Once the anodic current had returned to within $10 \mu \mathrm{A}$ of its steady value, the electrode was again switched to OC and held for $900 \mathrm{~s}$. The above procedure was repeated with varying OC times (Figure 6). During the open-circuit periods the cell potential was monitored with time as shown in Figure 6. Progressive removal of oxygen via Reaction (2) as predicted by the ChemicalElectrochemical mechanism is confirmed by the monotonic change in potential to more negative values with time. 


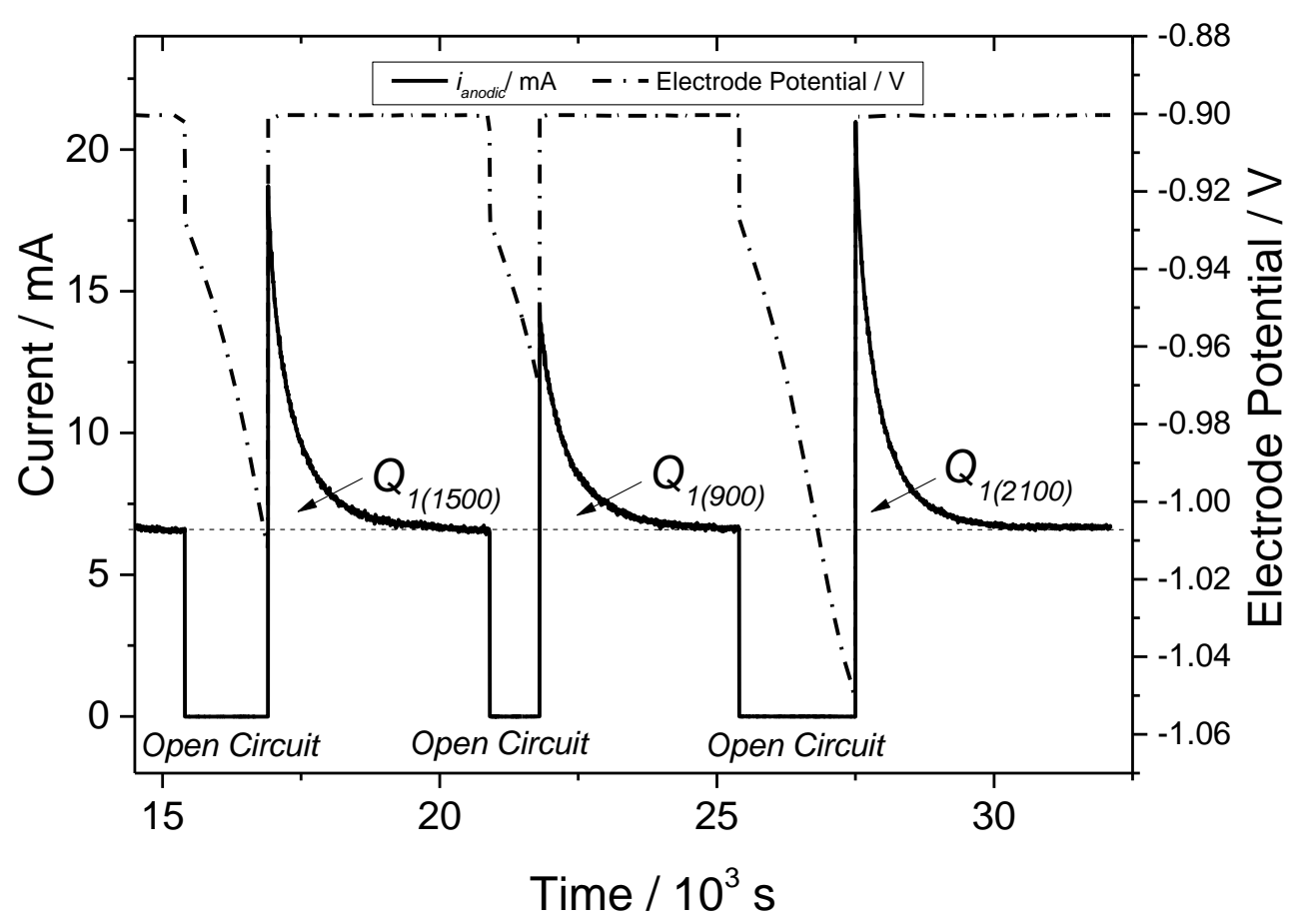

Figure 6. Operation of the working cell under Regime 1 with $16 \mathrm{kPa} p\left(\mathrm{H}_{2}\right)$ at $780{ }^{\circ} \mathrm{C}$.

The values of $Q_{1}$ were calculated by applying numerical integration to the area under the current-time curve (currents were measured at $5 \mathrm{~s}$ intervals) and are summarised in Table 1.

Table 1. $Q_{1}$ values obtained from with $16 \mathrm{kPa} p\left(\mathrm{H}_{2}\right)$ for Regime 1 operation.

\begin{tabular}{|l|c|c|c|}
\hline OC time / s & 900 & 1500 & 2100 \\
\hline$Q_{1}$ values / C & 3.98 & 5.35 & 5.71 \\
\hline
\end{tabular}

\section{2. $\quad$ Regime 2}

For Regime 2, consider a four-stage process (see Figure 7). Prior to A, a gaseous mixture of argon and hydrogen (3\%) was bubbled through liquid tin at open-circuit for a sufficient time to remove any residual oxide present in the melt. During the interval A $-\mathrm{B}$ the potential of the tin electrode is held at the potential $E$ and argon is flowed for sufficient time 
to reduce the current to a value close to zero. At B the tin electrode is then switched to opencircuit and the flowing gas to an argon-hydrogen mixture with hydrogen partial pressure $p_{2}^{\prime}$ for a period $t_{3}-t_{2}$. At $\mathrm{C}$ the flowing gas is returned to $100 \%$ argon and the potential of the electrode switched to the value $E$. The current is measured as a function of time for a period sufficient for the current to return to a value close to zero. The charge, $Q_{2}$, shown as the shaded area in Figure 7, is then calculated. Note that no hydrogen is bubbled into the tin during the period when the charge $Q_{2}$ is determined.

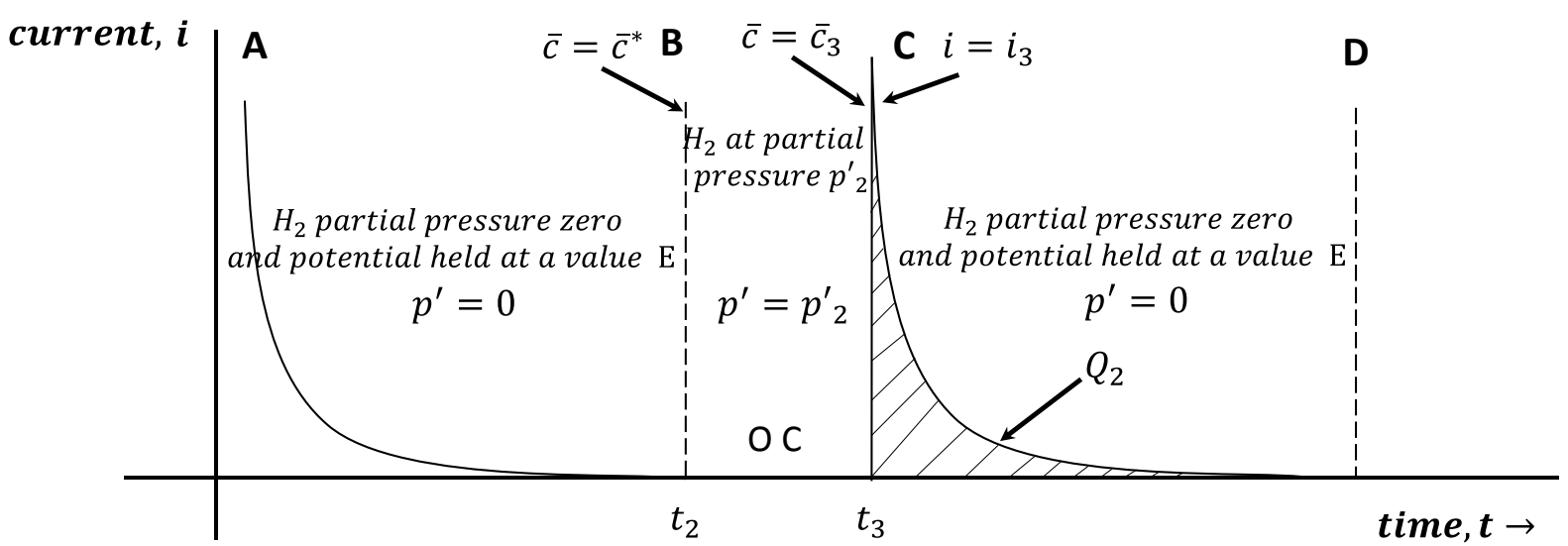

Figure 7. Schematic representation of Regime 2 conditions applied to the working cell flushed with constant flow of Ar.

Interval A-B does not require analysis in this treatment. For the interval B - C Equation (31) is used, replacing $\bar{c}_{2}$ by $\bar{c}^{*}$, (where $\bar{c}^{*}$ is the saturated concentration of oxygen at OC in the bulk of liquid tin at $p_{2}^{\prime}=0$ and potential $-0.90 \mathrm{~V}$ versus air reference):

$$
\int_{\bar{c}^{*}}^{\bar{c}_{3}} \frac{d \bar{c}}{\bar{c}}=-k_{1}\left(k_{2}\right)^{2} p_{2}{ }^{\prime} \int_{t_{2}}^{t_{3}} d t
$$

Then integrating

$$
[\ln \bar{c}]_{\bar{c}^{*}}^{\bar{c}_{3}}=-k_{1}\left(k_{2}\right)^{2} p_{2}{ }^{\prime}[t]_{t_{2}}^{t_{3}}
$$




$$
\begin{array}{r}
\ln \frac{\bar{c}_{3}}{\bar{c}^{*}}=-k_{1}\left(k_{2}\right)^{2} p_{2}{ }^{\prime}\left(t_{3}-t_{2}\right) \\
\bar{c}_{3}=\bar{c}^{*} \exp \left[-k_{1}\left(k_{2}\right)^{2} p_{2}{ }^{\prime}\left(t_{3}-t_{2}\right)\right]
\end{array}
$$

Region $\mathrm{C}-\mathrm{D}$ is now treated, where $p^{\prime}=0$. It follows that the charge, $Q_{2}$, shown in the diagram is given by:

$$
Q_{2}=\left(\bar{c}^{*}-\bar{c}_{3}\right) V n F
$$

Substituting for $\bar{c}_{3}$ from (41) into (42):

$$
Q_{2}=V n F \bar{c}^{*}\left\{1-\exp \left[-k_{1}\left(k_{2}\right)^{2} p_{2}{ }^{\prime}\left(t_{3}-t_{2}\right)\right]\right\}
$$

This is the required solution which may be compared with Equation (39).

\section{Corroborating experimental results in Regime 2}

In order to validate the above theory the LTA SOFC was operated in Regime 2. A mixture of $\mathrm{H}_{2}(16 \mathrm{kPa})$ and $\mathrm{Ar}$ was bubbled through the melt while the tin electrode was held at open circuit for $1500 \mathrm{~s}$. Then the tin electrode was switched from OC to a potential of -0.90 $\mathrm{V}$ vs. $\mathrm{RE}$ and the $\mathrm{H}_{2}$ was removed from the flow (argon continued to bubble into the tin). Once the measured anodic current (due to anodic injection of oxygen to replace that consumed by reaction with hydrogen) had reduced to zero, the electrode was switched to OC and remained so for $1500 \mathrm{~s}$ with flow of $16 \mathrm{kPa} p\left(\mathrm{H}_{2}\right)$ (Figure 8). The above procedure was repeated for OC intervals of 900 and 2100s. The flow of argon was constant throughout the experiment including during the open-circuit periods. As noted for Regime 1, progressive removal of oxygen via Reaction (2) is confirmed by the monotonic change in potential to more negative values with time (Figure 8). 


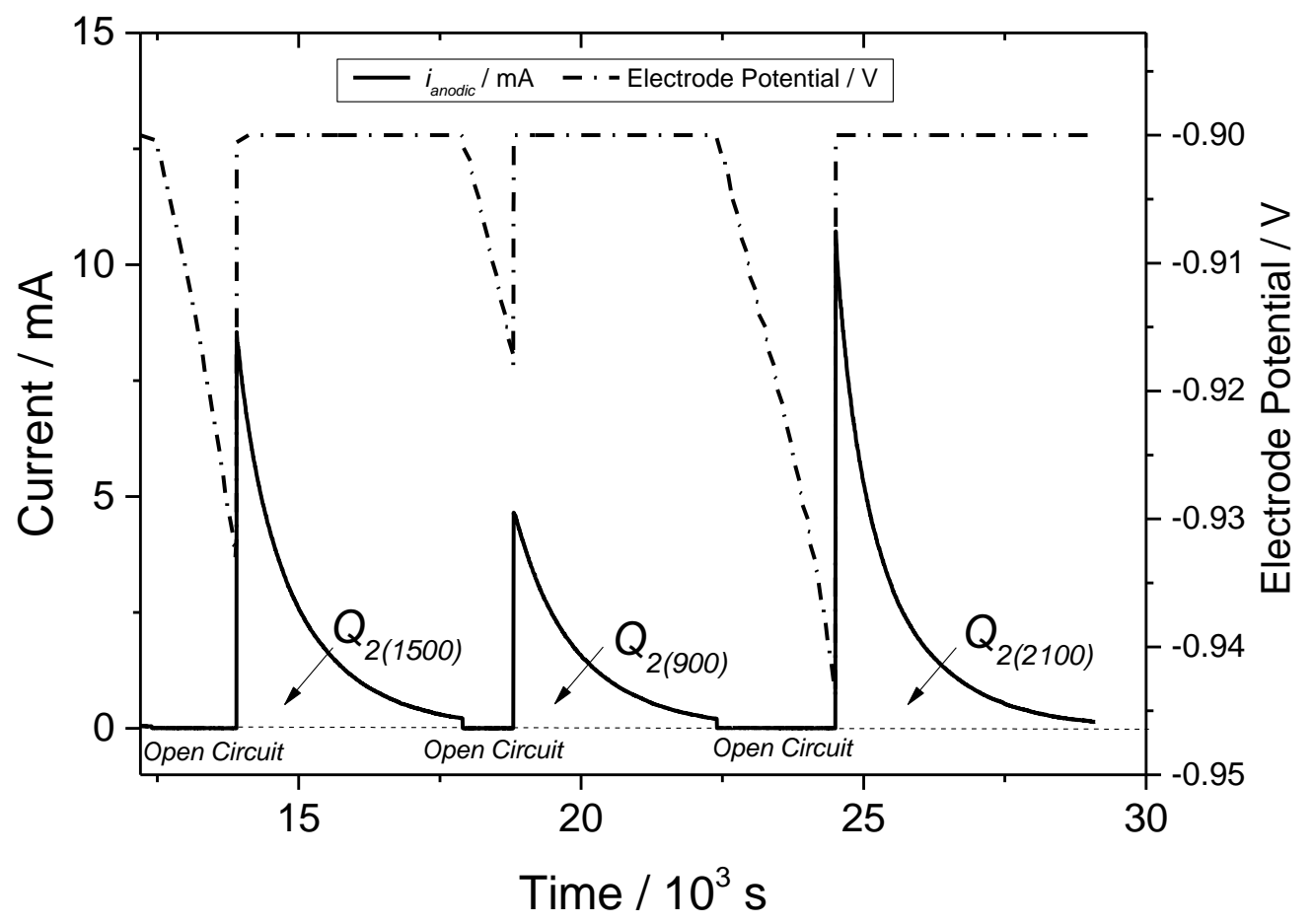

Figure 8. Operation of the working cell under Regime 2 with $16 \mathrm{kPa} p\left(\mathrm{H}_{2}\right)$ at $780{ }^{\circ} \mathrm{C}$.

The values of $Q_{2}$ were calculated in the same way as $Q_{1}$ values via numerical integration of the area under the current-time curve (currents were measured at $5 \mathrm{~s}$ intervals) and are summarised in Table 2.

Table 2. $Q_{2}$ values obtained with $16 \mathrm{kPa} p\left(\mathrm{H}_{2}\right)$ for Regime 2 operation.

\begin{tabular}{|l|l|l|l|}
\hline OC time / s & 900 & 1500 & 2100 \\
\hline$Q_{2}$ values / C & 5.11 & 8.05 & 8.90 \\
\hline
\end{tabular}

\section{3. $\quad$ Regime 3}

This case is included for completeness; however no experiments were performed to support the theory developed for Regime 3 as this is not required for determination of $\bar{z}$. This regime, which is a combination of the previous two regimes, is illustrated in Figure 9. 


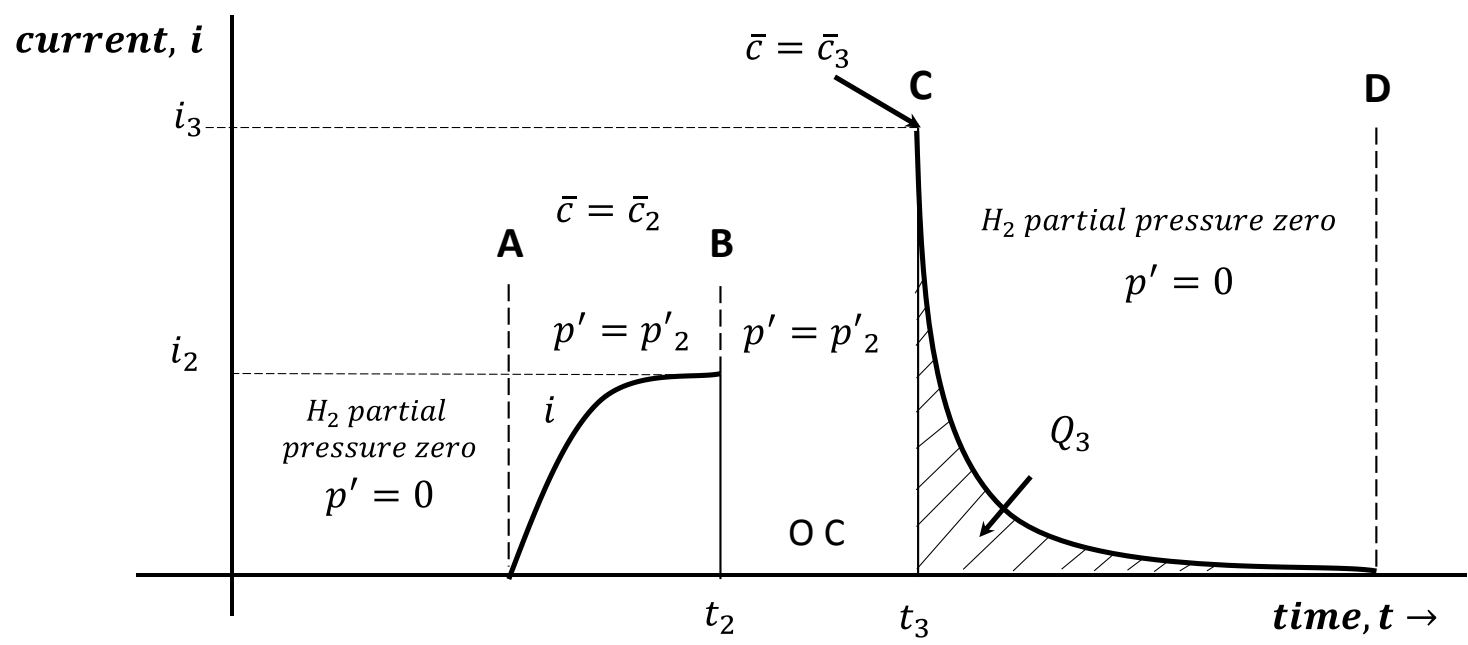

Figure 9. Schematic representation of Regime 3 conditions applied to the working cell flushed with constant flow of Ar; potential is held at a value $E$ everywhere except in B-C.

Prior to Point A, argon is bubbled through tin and the electrode is held at an anodic potential $E$, typically $-0.90 \mathrm{~V}$ with respect to an air electrode, while sufficient time is allowed for the current to reduce to a value close to zero. During the intervals $\mathrm{A}-\mathrm{B}$ and $\mathrm{B}-\mathrm{C}$, hydrogen at partial pressure $p_{2}{ }^{\prime}$ is bubbled through the tin. The interval $\mathrm{A}-\mathrm{B}$ (with potential maintained at $E$ ) is sufficiently long to allow the current to reach a steady value, $i_{2}$. During the interval B - C, of length $t_{3}-t_{2}$, the tin electrode is switched to open circuit. At Point $\mathrm{C}$ the flowing gas is returned to $100 \%$ argon and the tin electrode switched from OC to the potential $E$. Thereafter, the current is measured as a function of time until the current returns to a value close to zero. The charge $Q_{3}$ shown as the shaded area in Figure 9 is then calculated. Note that no hydrogen is bubbled into the tin during the period when the charge $Q_{3}$ is determined.

Following Regime 1, the oxygen concentration $\bar{c}_{3}$ at $\mathrm{C}$ is given by (32) which together with Equation (16) leads to:

$$
\bar{c}_{3}=\bar{z}_{2} \bar{c}^{*} \exp \left[-k_{1}\left(k_{2}\right)^{2} p_{2}^{\prime}\left(t_{3}-t_{2}\right)\right]
$$


For the interval $\mathrm{C}-\mathrm{D}$, as for Regime 2, the partial pressure of hydrogen is equal to zero (i.e. $p^{\prime}=0$ ). It follows that the charge, $Q_{3}$, shown in the diagram (Figure 9) is given by:

$$
Q_{3}=\left(\bar{c}^{*}-\bar{c}_{3}\right) V n F
$$

So from (44) and (45):

$$
Q_{3}=V n F \bar{c}^{*}\left\{1-\bar{z}_{2} \exp \left[-k_{1}\left(k_{2}\right)^{2} p_{2}{ }^{\prime}\left(t_{3}-t_{2}\right)\right]\right\}
$$

This is the required solution and may be compared with (39) and (43).

\section{Summary of the principal results in 3.1- 3.3}

The important equations in the above sections are those that allow interpretation of the Coulombic charge under the segment of each regime i.e. C - D. These are as follows:

Regime 1

$$
Q_{1}=\operatorname{VnF}\left(\bar{z}_{2}\right)^{2} \bar{c}^{*}\left\{1-\exp \left[-k_{1}\left(k_{2}\right)^{2} p_{2}{ }^{\prime}\left(t_{3}-t_{2}\right)\right]\right\}
$$

Regime 2

$$
Q_{2}=V n F \bar{c}^{*}\left\{1-\exp \left[-k_{1}\left(k_{2}\right)^{2} p_{2}{ }^{\prime}\left(t_{3}-t_{2}\right)\right]\right\}
$$

Regime 3

$$
Q_{3}=V n F \bar{c}^{*}\left\{1-\bar{z}_{2} \exp \left[-k_{1}\left(k_{2}\right)^{2} p_{2}{ }^{\prime}\left(t_{3}-t_{2}\right)\right]\right\}
$$

\subsection{Experimental determination of the Dynamic Oxygen Utilisation Coefficient, $\bar{z}$}

The development of a theoretical framework described earlier has identified the parameter $\bar{z}$. For a given hydrogen partial pressure, $p_{2}{ }^{\prime}$, and time period, $\left(t_{3}-t_{2}\right), \bar{z}$ may be determined using Equations (39) and (43) as follows:

$$
\left(\bar{z}_{2}\right)^{2}=\frac{Q_{1}}{Q_{2}}
$$


Taking the values of $Q_{1}$ and $Q_{2}$ from Table 1 and Table 2 respectively, the value of $\bar{z}_{2}$ from (47) is equal to 0.83 for a hydrogen partial pressure of $16 \mathrm{kPa}$.

\section{Conclusion}

Operation of a liquid metal anode SOFC fuelled with hydrogen via a ChemicalElectrochemical mechanism was investigated using the newly-proposed Anodic Injection Coulometry technique applied in two regimes of operation. The technique allows determination of the Dynamic Oxygen Utilisation Coefficient, $\bar{z}$, which has important implications with regard to design and operation of liquid metal anode SOFCs. The parameter $\bar{z}$ contains a new dimensionless number, which is similar to the Damköhler number. This dimensionless number contains geometric, mass transfer and kinetic factors that are dependent upon cell geometry and operating conditions. As an example of the application of this method, analysis of experimentally-generated data $\left(Q_{1}\right.$ and $Q_{2}$ values measured in Regimes 1 and 2, respectively) enabled a value of $\bar{z}$ to be evaluated for the specific geometry and operating conditions employed in this study.

\section{Acknowledgements}

The authors would like to thank Nazarbayev University and the Government of Republic of Kazakhstan for the BOLASHAK Scholarship for Dr. Aliya Toleuova and the EPSRC Supergen Fuel Cells programme (EP/G030995/1), Multiscale in-situ Characterisation of Degradation and Reactivity in Solid Oxide Fuel Cells (EP/J001007/1) and Electrodes by Design (EP/M014045/1) projects for supporting SOFC the research in the UCL Electrochemical Innovation Lab. Shearing recognises the Royal Academy of Engineering for 
support. We also acknowledge Prof. Ted Roberts (University of Alberta) for valuable technical discussion.

\section{References}

1. A. Toleuova, V. Yufit, S. Simons, W. C. Maskell, and D. J. L. Brett, J. Electrochem. Sci. Eng, 3, 91-105 (2013).

2. A. Jayakumar, R. Küngas, S. Roy, A. Javadekar, D. J. Buttrey, J. M. Vohs, and R. J. Gorte, Energy Environ. Sci., 4, 4133-4137 (2011).

3. A. Toleuova, W. C. Maskell, V. Yufit, P. R. Shearing, and D. J. Brett, J. Electrochem. Soc., 162, F988-F999 (2015).

4. H. M. Lee, Metall. Trans. A, 7A, 431-433 (1976).

5. C. Wagner, Thermodynamics of alloys, Addison-Wesley Press, (1952).

6. F. C. Campbell, Elements of Metallurgy and Engineering Alloys, AMS International, (2008), p. 105-106.

7. W. F. Hosford, Physical Metallurgy, CRC Press Taylor and Francis Group, Boca Raton, (2005), p. 36-37.

8. H. S. Fogler, Elements of Chemical Reaction Engineering, Fourth., Pearson Education, Upper Saddle River, NJ, (2006), p. 158.

9. M. J. Madou, Fundamentals of Microfabrication and Nanotechnology: Solid-State Physics, Fluidics and Analytical Techniques in Micro-and Nanotechnology, Third., CRC Press Taylor and Francis Group, Boca Raton, FL., (2011), p. 475.

10. G. B. Marin and G. S. Yablosnky, Kinetics of Chemical Reactions, Wiley-VCH Verlag \& Co. KGaA, Weinheim, (2011), p. 57.

11. T. R. Copeland and R. K. Skogerboe, Anal. Chem., 46, 1257-1268 (1974). 


\title{
Nomenclature
}

\author{
Parameters Description \\ and \\ Variables \\ $c^{\prime}$ \\ $\bar{c}$ \\ $\bar{c}^{*}$ \\ $\bar{c}_{1}$ \\ $\bar{c}_{2}$ \\ $\bar{c}_{3}$ \\ $\bar{D}$ \\ E \\ F \\ $\bar{J}$ \\ $i$ \\ $i_{2}$ \\ $i_{3}$ \\ $k_{1}$ \\ $k_{2}$ \\ $k_{3}$ \\ $\bar{N}$ \\ $p^{\prime}$ \\ $p_{1}^{\prime}$ \\ $p_{2}^{\prime}$ \\ $Q_{1}$ \\ $Q_{2}$ \\ $Q_{3}$ \\ $t$ \\ $t_{1}$ \\ $t_{2}$ \\ $c^{\prime}$ \\ Concentration of dissolved hydrogen in the bulk of liquid tin $/ \mathrm{mol} \mathrm{cm}$ \\ Concentration of dissolved oxygen in the bulk of liquid tin $/ \mathrm{mol} \mathrm{cm}{ }^{-3}$ \\ Saturated concentration of oxygen at OCin the bulk of liquid tin at $p^{\prime}=0 / \mathrm{mol} \mathrm{cm}^{-3}$ \\ Steady concentration of oxygen in the bulk of liquid tin at $p_{1}{ }_{1} \mathrm{~mol} \mathrm{~cm}^{-3}$ \\ Steady concentration of oxygen in the bulk of liquid tin at $p_{2} / \mathrm{mol} \mathrm{cm}^{-3}$ \\ Initial concentration of oxygen after switch from OC to potential, $E / \mathrm{mol} \mathrm{cm}^{-3}$ \\ Diffusion coefficient of oxygen in the melt $/ \mathrm{cm}^{2} \mathrm{~s}^{-1}$ \\ Applied potential / V \\ Faraday constant / 96,485 C mol-eq ${ }^{-1}$ \\ Flux of oxygen in the melt $/ \mathrm{mol} \mathrm{s}^{-1}$ \\ Anodic oxidation current / $\mathrm{mA}$ \\ Steady anodic current at $p_{2}^{\prime} / \mathrm{mA}$ \\ Initial (maximum) anodic current after switch from OC to potential, $E / \mathrm{mA}$ \\ Rate constant for homogeneous oxidation of hydrogen in liquid tin $/ \mathrm{s}^{-1} \mathrm{~mol}^{-2} \mathrm{~cm}^{6}$ \\ Equilibrium constant for hydrogen dissolution in liquid tin $/ \mathrm{molcm}^{-3} \mathrm{kPa}^{-0.5}$ \\ Proportionality constant / $\mathrm{C} \mathrm{cm}^{3}\left(\mathrm{~mol}_{-\mathrm{eq}^{-1}} \mathrm{~s}\right)^{-1}$ \\ Number of electrons transferred per hydrogen molecule \\ Number of moles of oxygen \\ Partial pressure of hydrogen supplied to the cell / $\mathrm{kPa}$ \\ Partial pressure of hydrogen supplied to the cell at $t=0 / \mathrm{kPa}$ \\ Partial pressure of hydrogen supplied to the cell at $t=t_{1} / \mathrm{kPa}$ \\ Coulombic charge in Regime 1(after switch of potential from OC to $E$ ) / C \\ Coulombic charge in Regime 2 (after switch of potential from OC to $E$ ) / C \\ Coulombic charge in Regime 3 (after switch of potential from OC to E) / C \\ Time / s \\ Time at which $p\left(\mathrm{H}_{2}\right)$ is changed from $p_{1}^{\prime}$ to $p_{2}^{\prime} / s$ \\ Time at which potential is switched from $E$ to $\mathrm{OC} / s$
}


$\bar{z}_{2}$

Superscripts

hydrogen 